\title{
Research on Correlation Between Psychological Factors, Mast Cells, and PAR-2 Signal Pathway in Irritable Bowel syndrome
}

This article was published in the following Dove Press journal: Journal of Inflammation Research

\section{Guanqun Chao' \\ Zhaojun Wang ${ }^{2}$ \\ Shuo Zhang $\mathbb{D}^{3}$}

'Department of General Practice, Sir Run Run Shaw Hospital, Zhejiang University, Hangzhou, People's Republic of China; ${ }^{2}$ The First Clinical Medical College of Zhejiang Chinese Medicine University, Hangzhou, People's Republic of China; ${ }^{3}$ Department of Gastroenterology, The First Affiliated Hospital, Zhejiang Chinese Medical University, Hangzhou, People's Republic of China
Correspondence: Shuo Zhang

Department of Gastroenterology, The

First Affiliated Hospital, Zhejiang Chinese

Medical University, Hangzhou, People's

Republic of China

Tel +86 I3957I92066

Email zhangshuotcm@163.com
Purpose: The study aimed to explore the level of psychological stress factors, mast cell (MC), and neuropeptide in the occurrence of irritable bowel syndrome (IBS) and the correlation among them, and to identify representative and effective indicators for the pathogenesis and clinical medication development of IBS.

Subjects and Methods: Twenty-eight patients (20-64 years old) with diarrheapredominant IBS (IBS-D) were included in the IBS-D group and 8 healthy subjects (35-63 years old) were enrolled in the control group. All subjects accepted the colonoscopic biopsies, self-rating depression scale (SDS) and self-rating anxiety scale (SAS) assessment. MC degranulation, neuropeptide S (NPS), neuropeptide Y (NPY), NPY receptor 2 (NPY2R) and Protease-activated receptor 2 (PAR-2) in colon tissues were performed by Strept Avidin-Biot complex (SABC) immunohistochemistry. Enzyme-linked immunosorbent assay (ELISA) detection was used to test the expression of NPS and NPY in peripheral blood plasma and colon tissues. Western blot was applied to examine the level of NPY2R and PAR-2.

Results: The level of anxiety and depression of patients with IBS-D was more serious than that in the control. The expression of NPS, NPY and NPY2R was down-regulated in the IBS$\mathrm{D}$. The total MC and tryptase-positive MC increased significantly in the colon tissue of IBS$\mathrm{D}$ and the expression level of PAR-2 was significantly up-regulated.

Conclusion: There has been a close connection among those indicators that the activated MC may up-regulate the function of PAR-2, resulting in the change of neuropeptide (NPS and NPY), successively leading to clinical symptoms and psychological negative changes in the IBS.

Keywords: Irritable bowel syndrome, diarrhea, neuropeptide, protease-activated receptor 2, mast cell

\section{Background}

Irritable bowel syndrome (IBS) is a common disease with a high incidence rate that imposes enormous burdens on healthcare systems. ${ }^{1}$ According to the predominant stool form and bowel habits of patients there are four heterogeneous clinical phenotypes of IBS: diarrhea-predominant IBS (IBS-D), constipation-predominant IBS (IBS-C), mixed IBS and unclassifiable IBS. ${ }^{2}$ IBS features various symptom combinations including abdominal pain mainly, bloating, and altered stool frequency, in the absence of any detectable organic disease or available clinical tests and examinations. ${ }^{3}$ It has been reported that the worldwide prevalence of IBS is 
approximately $10-20 \%{ }^{4}$ The pathogenesis of IBS is unclear so far, however, multifactorial factors such as genetics, dietary intolerance, alterations in the gastrointestinal (GI) microbiota, visceral hypersensitivity, increased intestinal permeability, small intestinal overgrowth (SIBO), intestinal immune activation, disruption of the gut-brain axis, abnormal pain processing, behavioral pathways, and altered GI motility have been reported. ${ }^{5,6}$

Comorbidity of IBS and psychological disorders is known to be widespread. According to US statistics, $47 \%$ of patients with IBS have a variety of common mental diseases, including major depression, generalized anxiety disorder, panic disorder, somatization disorder and post-traumatic stress disorder, etc. ${ }^{7}$ Studies also confirmed that psychological stress can increase the visceral sensitivity in the gastrointestinal tract, thus playing an important role in the onset of functional gastrointestinal diseases such as IBS. ${ }^{8}$ What's more, other researches further revealed that mental anxiety and depression, which may be regulated by some major tyrosine peptides, such as Neuropeptide Y (NPY), ${ }^{9}$ were closely associated with the onset of IBS-D and can be an effective target for its treatment. $^{10}$ In fact antidepressants, antianxiety drugs, and some other psychotherapy (non-drug therapy) do exert exact effects on IBS to a certain extent. ${ }^{11}$

Neuropeptide S (NPS), a 20 amino acid peptide, which is highly conserved among mammals, works by binding to the NPS receptor selectively. ${ }^{12}$ The activation of the NPS system can lead to relief of anxiety. ${ }^{13}$ Another neuropeptide, NPY, as a 36-residue amidated peptide, is simultaneously existed in the central and peripheral nervous systems. ${ }^{14}$ Combining with the receptor, the NPY system can control memory retention, energy homeostasis, sleep regulation, regulation of food intake and vasoconstriction. Applied for the development of various kinds of agonists and antagonists, it plays a critical role in treating diseases such as epilepsy, neurodegenerative disorders, obesity, and psychiatric disorders. ${ }^{15}$ Protease-activated receptor 2 (PAR-2), also called trypsin receptor 1, belongs to a family of four $G$ protein-coupled receptors, which is reported to be connected with visceral pain in the colon and results in increased recruitment of inflammatory cells, including mast cell (MC). ${ }^{16,17}$ Inflammatory molecules secreted by those activated cells, such as histamine and proteases, increase the synthesis of inflammatory mediators by $\mathrm{MC}$, which promote visceral hypersensitivity in patients with IBS-D. ${ }^{18}$ Abnormal activation of PAR-2 by colonic $\mathrm{MC}$, in turn, appears to induce visceral hypersensitivity in patients with IBS-D as well. So far accumulated pieces of evidence have indicated that IBS pathophysiology has been linked to life stress, epithelial barrier dysfunction, and MC activation. ${ }^{19}$ Yet the role of mediators of stress responses in the gastrointestinal tract on IBS mucosal function remains largely unknown. Therefore, we hypothesize that there might be a linked relationship between MC, PAR-2 and neuropeptide, which could cause relevant clinical symptoms and psychological negative changes in IBS patients.

This trial selected IBS-D patients, with the assessment of depression and anxiety, measuring the expression level of NPS, NPY, PAR-2, NPY2R, exploring the correlation of psychological stress, MC, PAR-2 and brain intestinal peptide in the occurrence and development of IBS, actively investigating representative and effective indicators for pathophysiology and clinical treatment of IBS.

\section{Subjects and Methods Subjects}

This trial was approved by the ethics committee of the Sir Run Run Shaw Hospital. Colonic mucosal biopsy specimens were collected with the informed consent of patients. The whole process of this study was conducted in accordance with the Declaration of Helsinki.

From October 1, 2016 to September 30, 2017, 28 patients who met the IBS-D inclusion criteria were recruited, including 13 males and 15 females, aged from 20 to 64 years old with an average age of 44.35 years. While simultaneously 8 healthy volunteers were enrolled as normal control, including 4 males and 4 females, aged from 35 to 63 years, with a mean age of 47.88 years. All subjects included in the study were informed of relevant matters and signed informed consents.

\section{IBS Diagnostic Standard}

The diagnostic criteria for IBS-D patients were derived from Roman IV standards: In the last three months, at least 3 days per month of recurrent abdominal pain, and with the two or more items described below: abdominal pain and bowel movement; Frequency of defecation changes ( $>3$ times per day); Fecal changes (watery stool or mucus stool).

Symptoms of IBS-D patients appear at least 6 months prior to the establishment of the diagnosis and last for 3 months. And abdominal pain occurs at least one day a week. Importantly, there is no evidence of organic 
lesions that could explain the above symptoms, including biochemical and B-ultrasonography inspection. Only patients who meet all the above criteria can be finally diagnosed with IBS-D.

\section{Inclusion Criteria}

Subjects conformed to all of the following claims were included in the IBS-D group: 1) Patients who meet the above IBS-D diagnose standard; 2) No evidence of structural diseases that can explain the above symptoms (including biochemical, gastrointestinal endoscopy, b-ultrasonography, etc.); 3) Age from 18 to 70 years old; 4) Superior informed consent and signed informed consent.

Recruited healthy control volunteers should qualify the conditions below: 1) Age from 18 to 70 years old; 2) No history of digestive diseases; 3 ) Normal physical examination, colonoscopy, B-ultrasonography and other examinations; 4) No recent history of medication.

\section{Exclusion Criteria}

Subjects with any of the following conditions will be excluded: 1) Not in the included age range; 2) Accompanied by acute intestinal infection; 3) Diagnosed as organic lesions such as enteritis, peptic ulcer, gastrointestinal bleeding, and digestive tract tumor; 4) Individual with mental illness or unable to describe subjective symptoms of discomfort independently; 5) Suffering from severe cardiovascular, cerebrovascular, hepatorenal, endocrine and hematopoietic diseases; 6) With a history of abdominal surgery; 7) Taking anticholinergic and nonsteroidal anti-inflammatory drugs and hormone drugs; 8) Participating in other trials in the last six months; 9) Pregnant or breastfeeding.

\section{Methods}

\section{Mental Health Assessment}

Self-rating anxiety scale (SAS) and self-rating depression scale (SDS) were applied to evaluate the mental emotion of subjects in both groups. Score collection and counting were executed by researchers blinded to the grouping. The boundary-value of the SAS scale anxiety state was 50 points, and the boundary-value of the SDS scale depression state was 53 points. The higher the score was, the more serious the anxiety or depression situation was. Analysis of scores was used to compare the difference of mental state between the healthy control and the IBS group.

\section{Specimen Collection and Disposal}

Fasting for 6 to 8 hours, $4 \mathrm{~mL}$ of venous blood specimens was collected (medical EDTA tube), kept for 30 minutes and then followed by centrifugation for $10 \mathrm{~min}$ at a speed of $3000 \mathrm{~g}$. Transferring supernatant fluid to the tube and numbering. The prepared sample to be tested could be stored in a $-80^{\circ} \mathrm{C}$ refrigerator for later use. All subjects underwent a colonoscopy after adequate bowel preparation. And the uniform types of biopsy forceps were applied for sampling. Three mucosal biopsies were drawn from the ileocecal region ascending colon about $5 \mathrm{~cm}$ away from the distal end of the ileocecal valve opening during colonoscopy.

Blocks were washed and dehydrated in ascending series of ethanol, fixed in $4 \%$ formalin for $24 \mathrm{~h}$ and embedded routinely with paraffin, every section was 3 to $5 \mu \mathrm{m}$. Have sections conventional hematoxylin-eosin (HE) stained, then histopathological changes were observed by Olympus light microscope (x 400).

\section{Mast Cell (MC) Degranulation Was Detected by SABC}

Strept Avidin-Biotin Complex (SABC) assay, a modified immunohistochemical (IHC) technique, ${ }^{20}$ was used to detect tryptase-positive MC. ${ }^{21}$ SABC-Pod Kit (Solarbio, Beijing, China) was performed in the procedure. Briefly, the paraffin section was dewaxed and rehydrated. Peroxidase activity was quenched by $3 \% \mathrm{H}_{2} \mathrm{O}_{2}$ for 10 min and then washed with PBS (5 min, 3 times). After washing the sample with tris buffer, the first and biotinylated second antibody were added successively to incubate for $60 \mathrm{~min}$ and $20 \mathrm{~min}$, respectively, at the constant temperature of $37^{\circ} \mathrm{C}$. Then undergoing the procedures of rinsing, SABC-Pod reagent incubation, and diaminobenzidine tetrahydrochloride (DAB) staining, etc. All specimens were counterstained with Macklin's Hematoxylin, examined and photographed on an Olympus microscope, 3 high-power fields (x 400) were randomly selected for MC count (MCC).

Refer to previous research, ${ }^{22} \mathrm{MC}$ were identified by their metachromatic cytoplasmic granules. Its degranulation was assessed based on unclear or irregular cell membranes and the presence of extruded secretory granules (tryptase-positive). The average MC number per millimeter square of the mucosal area was counted. The ratio of degranulated $\mathrm{MC}$ was also calculated in each section (degranulated $\mathrm{MC} /$ the total number of $\mathrm{MC} \times 100 \%$ ). And the quantitative analysis was made by using the Image-Pro 
Plus 6.0 software (Media Cybernetics, Inc. US), which depends on the average integrated optical density (IOD). During the analysis, the average IOD of positive staining substances of three non-overlapping fields chosen randomly was measured for the final analysis.

\section{ELISA}

Quantitative determination of NPS and NPY in peripheral blood plasma and colon tissues were detected by enzymelinked immunosorbent assay (ELISA). The procedure was performed by referring to relevant previous literature ${ }^{23}$ and manufacturer's instructions of Human NPS/NPY ELISA kit (FineTest, Wuhan, China).

\section{Colon Tissue Immunohistochemistry SABC}

About 5 - $\mu \mathrm{m}$-thick continuous paraffin sections were prepared from selected colon tissue. Expression of NPS, NPY, NPY2R and par-2 in colon tissues was detected by the SABC IHC method. The specific processed steps referred to the previous study ${ }^{24}$ and SABC Kit instructions (Solarbio, Beijing, China): xylene dewaxing, gradient alcohol hydration, $0.3 \% \mathrm{H}_{2} \mathrm{O}_{2}$ blocking endogenous peroxidase, rising, incubation with primary antibody and then with standard labeled streptavidin antibody biotin, washing and developing in DAB hydrochloride.

\section{Western Blot}

The Western blot method was adopted to detect quantitative levels of NPY2R and PAR-2. NPY2R antibody (GeneTex, Texas, USA) and PAR-2 antibody (FineTest, Wuhan, China) were used in the procedure. To prepare for total protein extraction, the tissues were grinded with liquid nitrogen, homogenized in lysis buffer and protease inhibitors (Sigma, MO, USA), followed by centrifugation at 10,000 g for $20 \mathrm{~min}$. Then the collected supernatant was loaded on the prepared sepharose gel for electrophoresis (Bio-RAD, USA) and later transferred to the PVDF membrane (Millipore, USA) for electric rotation, with the parameter of $200 \mathrm{~mA}$ current for 90 minutes. After being taken out, the membrane was placed into the blocking solution, subsequently incubated with antibodies twice. Finally, the cleaned membrane was placed on the preservative film for titration, the chemiluminescence method was used for detection, and images were collected for analysis. ImagePro Plus 6.0 software (Media Cybernetics, Inc. US) was used for measuring the gray level to quantify the expression of NPY2R and PAR-2.

\section{Statistical Analysis}

Graph Prism 6.0 software (GraphPad Prism, San Diego, CA, USA) was used for image analysis. The prophase data were processed by Microsoft Excel and later statistically analyzed by SPSS 17.0 software package (SPSS Inc., Chicago, IL, USA). Counting data were checked by chisquare test, while the mean number comparisons between the measurement data group in the normal state were performed by $t$-test, and the non-parametric test was used for non-normal data. $p<0.05$ and $p<0.01$ were considered as statistically significant and very significant, respectively.

\section{Results}

Twenty-eight cases were included in the IBS-D group, 15 cases for females and 13 cases for males, with an age range from 20 to 64 years old and the mean age of 44 years old $(44.46 \pm 11.90)$. Eight cases were enrolled in the control group, aged from 35 to 63 years old, with an average age of 48 years old $(47.88 \pm 9.82)$ and a sex ratio $M \backslash F=1: 1$. There was no statistically significant difference in gender and age between the two groups $(p>0.05)$.

\section{Anxiety and Depression Difference}

Among all 28 patients with IBS-D, 9 cases had anxiety alone, 6 cases had depression simply, and 3 cases suffered from both anxiety and depression. Those 18 patients with comorbid anxiety and/or depression included 11 patients with mild anxiety, 1 patient with moderate anxiety, and 9 patients with mild depression. Thus, $64.29 \%$ (18/28) of patients with IBS-D were accompanied by anxiety and/or depression to varying degrees. The analysis result of the SAS score was $48.29 \pm 1.05$, and the SDS score was 49.36 \pm 0.77 .

Among 8 healthy controls, there were two individuals suffered mild anxiety and mild depression, respectively. The SAS score $(42.50 \pm 1.90)$ and the SDS score $(43.75 \pm$ $1.54)$ of the control group were significantly lower than that in the IBS-D group $(p=0.013, p=0.002)$.

\section{Histopathological Changes}

As shown in Figure 1, healthy individuals had normal columnar villi in their colon tissues, the mucosal epithelial cells and mucosal intrinsic layer cells were arranged in a neat sequence with regular clear boundaries, normal MC and non-obvious inflammatory cells (Figure 1A). The columnar mucosal 

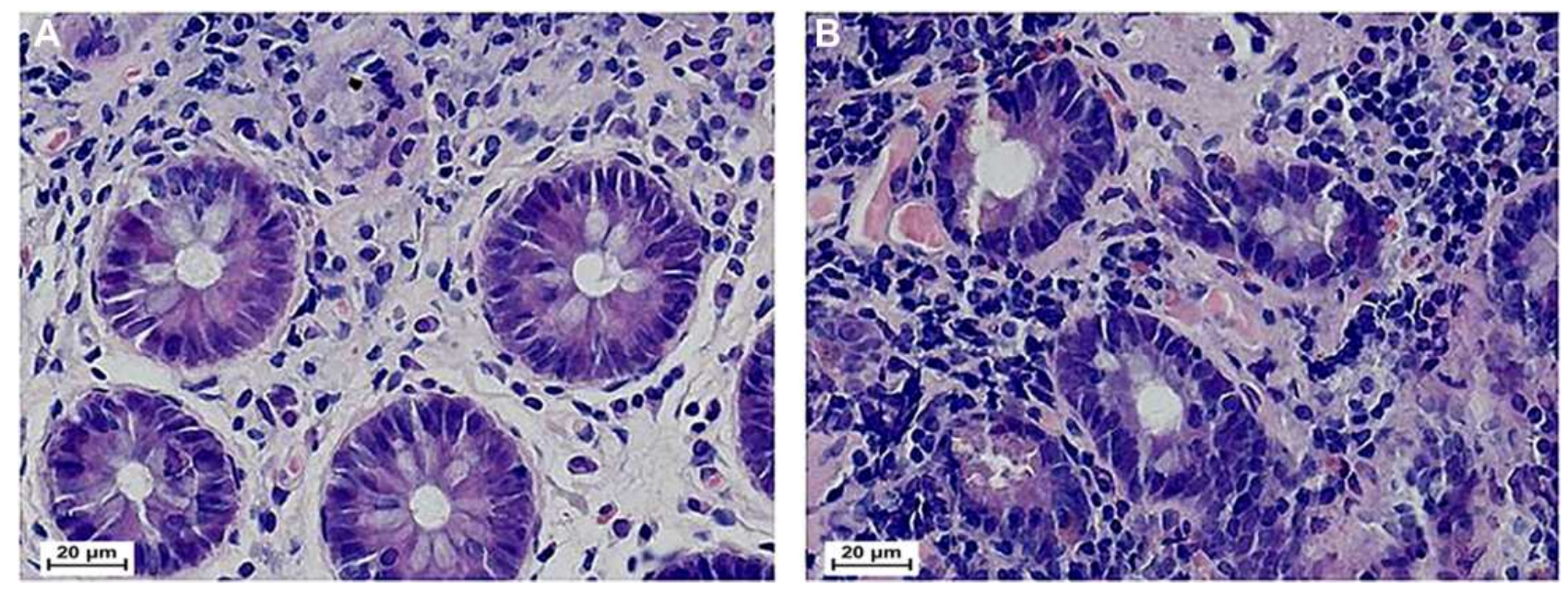

Figure I Histopathological changes of the two group $(\times 400)$. (A) Colonic mucosal histology of normal controls $(\times 400)$; (B) Colonic mucosal histology of IBS-D patients $(\times$ 400). The positive cells were purple and blue. Hematoxylin-eosin (HE) was used for staining in the detection. IBS-D: Diarrhea-predominant irritable bowel syndrome.

structure of colon tissue was basically normal in patients with IBS-D. Partial mucosal epithelial cells were irregular, the intrinsic mucosal boundaries were obscure, with a massive increase in $\mathrm{MC}$ and inflammatory cells in the space (Figure 1B).

\section{Mast Cell Degranulation}

Compared with the control group $(0.25 \pm 0.05)$, the mean IOD of tryptase-positive MC increased significantly ( $p<$ $0.05)$ in the colon tissue of the IBS-D $(0.40 \pm 0.07)$. The stained brown tryptase-positive MC in the IBS-D patient (Figure 2B) was significantly more than that in the normal control (Figure 2A). And the percentage of MC degranulation was significantly increased $(p<0.01)$ in the colon tissue of the IBS-D $(42.4 \pm 6.51 \%)$ than that in the control $(22.7 \pm 4.00 \%)$.

\section{Colon Tissue SABC Immunohistochemistry}

Results showed that the average IOD value of NPY2R in the IBS-D $(0.24 \pm 0.05)$ decreased significantly $(p<0.05)$ compared with the healthy colon tissue $(0.35 \pm 0.07)$. The average IOD value of PAR-2 in the IBS-D $(0.60 \pm 0.06)$ increased significantly $(p<0.05)$ compared with healthy colon tissue $(0.48 \pm 0.05)$. The mean IOD of NPS in the colon of patients with IBS-D was $0.29 \pm 0.06$, and it was significantly lower than the positive expression level of the healthy control group $(0.41 \pm 0.03)$, the difference
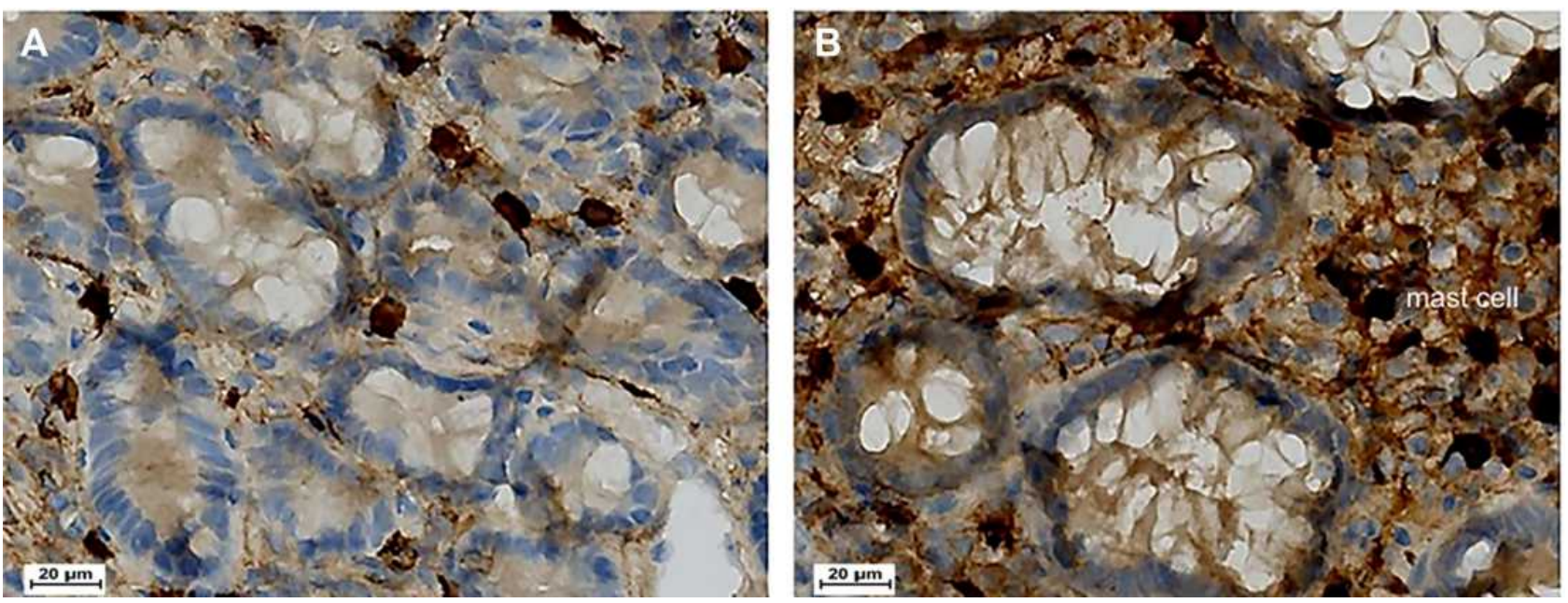

Figure 2 Immunohistochemical results of tryptase-positive MC ( $\times 400)$. (A): Tryptase-positive mast cell $(M C)$ in the normal subject $(\times 400)$; $(\mathbf{B})$ Tryptase-positive MC in the patient with IBS-D $(\times 400)$. The positive cells manifested brown or yellow stained. 
was statistically significant $(p=0.001)$. The mean IOD of NPY in the colon of patients with IBS-D was $0.38 \pm 0.10$, and it was significantly lower than the positive expression level of the healthy control group $(0.51 \pm 0.12)$, the difference was statistically significant $(p=0.016)$. The specific distributions of the four substances in the two groups were shown in the immunohistochemical images (Figure 3A-H).
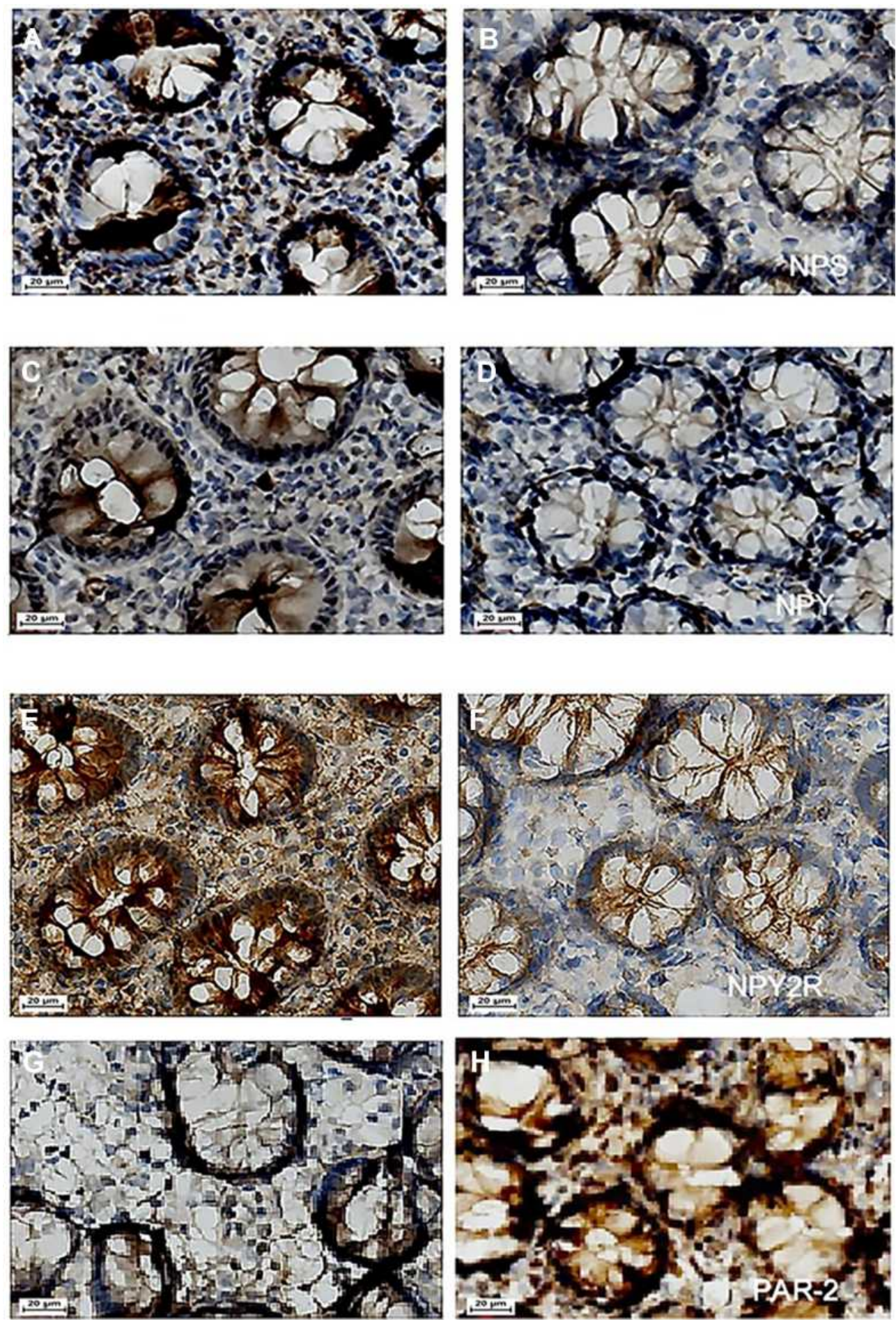

Figure 3 Immunohistochemical results of NPS, NPY, NPY2R and PAR-2 $(\times 400)$. (A) NPS in the normal group $(\times 400) ;(B)$ NPS in the IBS-D group $(\times 400)$; (C) NPY in the normal group; (D) NPY in the IBS-D group ( $\times 400)$; (E) NPY2R in the normal group $(\times 400)$; (F) NPY2R in the IBS-D group $(\times 400)$; (G) PAR-2 in the normal group $(\times 400)$; (H) PAR-2 in the IBS-D group $(\times 400)$.

Abbreviations: NPS, Neuropeptide S; NPY, Neuropeptide Y; NPY2R, NPY receptor 2; PAR-2, Protease-activated receptor 2. 


\section{Expression of NPS and NPY by ELISA}

Respectively, the content of NPS and NPY in the peripheral blood plasma of patients with IBS-D was 8.50 $\pm 2.72 \mathrm{ng} / \mathrm{L}$ and $260.85 \pm 76.21 \mathrm{pg} / \mathrm{mL}$. Both significantly lower than that in the healthy control group (NPS [11.80 $\pm 3.88 \mathrm{ng} / \mathrm{L}]$, NPY $[511.27 \pm 143.95 \mathrm{pg} / \mathrm{mL}])$ the difference was statistically significant $(p=0.025, p=0.011$, respectively).

The levels of NPS and NPY in the colon tissue of patients with IBS-D were $8.47 \pm 1.51 \mathrm{ng} / \mathrm{L}$ and 316.59 $\pm 78.50 \mathrm{pg} / \mathrm{mL}$, respectively. Both significantly lower than that in the healthy control (NPS [13.85 \pm 6.08 $\mathrm{ng} / \mathrm{L}]$, NPY $[539.19 \pm 276.78 \mathrm{pg} / \mathrm{mL}])$, the difference was statistically significant $(p=0.027, p=0.001$, respectively).

\section{Expression of NPY2R and PAR-2 by Western Blot}

Compared with the normal group $(1.00 \pm 0.24)$, the relative expression level of NPY2R protein in colon tissues of patients with IBS-D $(0.59 \pm 0.15)$ was significantly decreased $(p<0.05)$. And the relative expression level of PAR-2 protein in the IBS-D $(1.67 \pm 0.33)$ was significantly increased $(p<0.05)$ than that in the control $(1.00 \pm 0.37)$

(Figure 4A-C).

\section{Discussion}

A tremendous amount of studies have shown that psychological stress such as anxiety and depression play an important role in the pathogenesis of IBS. Gopi et $\mathrm{al}^{25}$ even found that anxiety and depression scores of patients

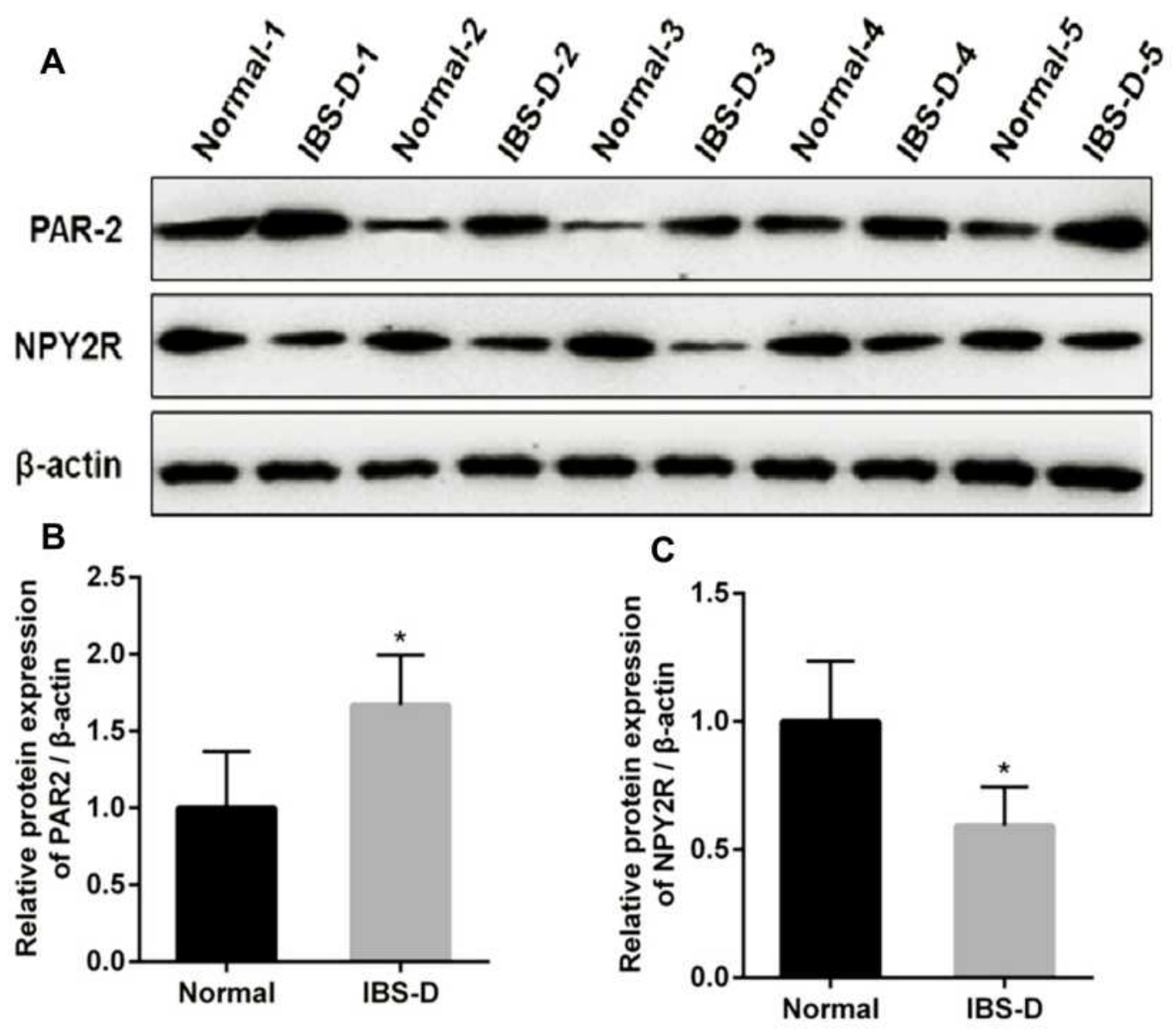

Figure 4 Expression of PAR-2 and NPY2R in the two group. (A) NPY2R and PAR-2 protein bands detected by Western blot; (B) The expression of PAR-2 in the IBS-D group was significantly higher than that in the normal group; (C) The expression of NPY2R in the IBS-D group was significantly lower than that in the normal group. *represents $p<0.05$ vs Normal.

Abbreviations: NPY2R, NPY receptor 2; PAR-2, Protease-activated receptor 2. 
with IBS-D were higher than those with IBS-C. Elyse et $\mathrm{al}^{26}$ also confirmed that the risk of psychological disorders in IBS patients increased significantly, and the living quality of those patients was negatively correlated with anxiety and depression. Same as previous studies, our study demonstrated a higher level of anxiety and depression in IBS-D patients as well.

As regards the mechanism of IBS, the brain-gut axis and ghrelin are catching more scholars' attention. Dysfunction of the neuroendocrine network two-way loop channel in the brain-gut axis is widely regarded as a key point in the pathophysiology of IBS. ${ }^{27}$ As an important neuropeptide in the axis, NPS has the function of adjusting anxiety, influencing feeding behavior, reducing panic attacks, regulating the inflammatory response, immune response, hurt feelings and allergy effect, by binding with its receptor. ${ }^{28} \mathrm{~A}$ multicenter study that included 1664 patients with bowel disease found that the incidence was related to the diversity of NPS receptor genes. ${ }^{29}$ Experimental study on mice also shown that NPS injection into the main center inhibited the distal colon transmission, ${ }^{30}$ and injection into the lateral ventricle inhibited the excretion of feces, while inhibitor injection into the lateral ventricle simultaneously antagonized the inhibition of the colon movement caused by NPS. ${ }^{29}$ It can be seen that NPS is closely related to emotion and intestinal symptoms both.

Existing in the submucosal nerve mainly, NPY functions as an important neurotransmitter in the gastrointestinal tract, regulating the secretion of and absorption, and adjusting the pressure by affecting the hippocampus and hypothalamus and emotions. ${ }^{29}$ Review pointed out that underlying NPY system-related neurobiological factors contributed to the comorbidity of stress disorder and gastrointestinal disorders such as IBS, and even NPY levels in bowel tissue are lower in IBS-D versus IBS-C. ${ }^{31}$ Research in Australia found that NPY inhibited colon transportation. ${ }^{32}$ It indicated that NPY is negatively correlated with emotions (such as tension and pessimism) and gastrointestinal movement. ${ }^{33}$ This is in agreement with the results of our study. That's to say, psychological stress factors disturb peptide secretion in the brain-gut axis, resulting in the reduction of NPY and NPS, successively causing the enhanced movement and secretion of the bowel in the IBS-D. Thus, NPY and NPS may be the key node of gastrointestinal dysfunction caused by psychological stress.
Intestinal mucosal mast cells (IMMC), existing in the intrinsic layer of the intestinal mucosa, are known as the fourth barrier of the gastrointestinal mucosa. ${ }^{34}$ It exhibits important effects on GI pathophysiology and physiology, particularly in secretion, intestinal sensation, permeability, motility, and inflammation. ${ }^{35}$ Research demonstrated that biopsies from patients with IBS had larger numbers of MCs, a higher percentage of MCs, higher levels of tryptase $^{36}$ (the product of MC degranulation, which is considered as the mark of activated MC). Some studies identified that tryptase had a positive effect on PAR- $2,{ }^{37}$ which was reported to be linked to visceral pain in the colon. ${ }^{38}$ Our findings verified the same changing trends, with enhanced IOD of tryptase-positive MC, increased proportion of MC degranulation and up-regulated level of PAR-2. Besides, further research reported that psychological stress is also one of the factors to activate intestinal $\mathrm{MC},{ }^{35} \mathrm{MC}$ degranulated tryptase influence neuronal signaling by activating pro-inflammatory and PAR-2 pathways. ${ }^{39}$ Therefore, we speculate that not only the low level of neuropeptide but also the activation of MC as well as PAR-2 may be responsible for the anxiety and/ or depression scores and symptoms of the IBS-D.

The limitations of the study are the small sample size of patients and focus on only part of the colon and protein level of the indicators without animal experimental verification. Further research targeting the mast cell-PAR-2 signaling is supposed to carry out a basic confirmatory test with agonists or antagonists, identifying the specific mechanism network and investigating therapeutic measures against IBS-D.

\section{Conclusion}

IBS-D patients are more likely to suffer from psychological disorders, which in turn induce or aggravate the clinical symptoms of IBS. There might be a close connection among the activated MC, increased PAR-2, and reduced NPY and NPS in the IBS-D accompanied by anxiety or depression: the activated MC may up-regulate the function of PAR-2, resulting in the change of neuropeptide (NPS and NPY), successively leading to clinical symptoms and psychological negative changes in the IBS-D. That may be the worthwhile targets for IBS therapy.

\section{Abbreviations}

IBS, irritable bowel syndrome; IBS-D, diarrheapredominant IBS; GI, gastrointestinal; SAS, self-rating anxiety scale; SDS, self-rating depression scale; MC, mast cell; NPY2R, NPY receptor 2; PAR-2, protease-activated 
receptor 2; NPS, neuropeptide S; NPY, neuropeptide Y; ELISA, enzyme-linked immunosorbent assay; SABC, strept avidin-biotin complex; IHC, immunohistochemistry; IOD, integrated optical density; SIBO, small intestinal overgrowth; IMMC, intestinal mucosal mast cells.

\section{Ethics Approval and Informed Consent}

This trial had been approved by the ethics committee of Sir Run Run Shaw hospital. Informed consent of each patient had also been acquired. The whole process of this study was conducted in accordance with the Declaration of Helsinki.

\section{Author Contributions}

Shuo Zhang had a leading role in the conception, study design and execution, meanwhile, assisted in drafting the article for important intellectual content. Guanqun Chao participated in the design, implemented the experiment, did the analysis or interpretation, and written the manuscript. Zhaojun Wang took part in planning, completed the acquisition and explanations of data, substantially revised the article. All authors agreed to submit to the current journal, gave final approval of the version to be published, and agreed to be accountable for all aspects of the work.

\section{Funding}

This research was supported by the National Natural Science Foundation of China (82074214, 81973598, 81573760), Zhejiang Provincial Natural Science Foundation of China (LY18H030001, LY16H030010), Medicine and Health Science and Technology Plan Projects in Zhejiang province (2017KY413), Traditional Chinese Medicine Science and Technology Plan of Zhejiang Province (2017ZA089, 2016ZB071, 2015ZZ012, 2014ZA030) and Medical Health Platform Plan Projects of Zhejiang Province (2015RCA020).

\section{Disclosure}

The authors have no conflicts of interest in this work.

\section{References}

1. Sperber AD, Dumitrascu D, Fukudo S, et al. The global prevalence of IBS in adults remains elusive due to the heterogeneity of studies: a Rome foundation working team literature review. Gut. 2017;66 (6):1075-1082. doi:10.1136/gutjnl-2015-311240

2. Lacy BE, Mearin F, Chang L, et al. Bowel disorders. J Gastroenterol. 2016;150(6):1393-1407. doi:10.1053/j.gastro.2016.02.031
3. Chey WD, Kurlander J, Eswaran S. Irritable bowel syndrome: a clinical review. JAMA. 2015;313(9):949-958. doi:10.1001/ jama.2015.0954

4. Thompson WG, Heaton KW, Smyth GT, Smyth C. Irritable bowel syndrome in general practice: prevalence, characteristics, and referral. Gut. 2000;46:78-82. doi:10.1136/gut.46.1.78

5. Bolino CM, Bercik P. Pathogenic factors involved in the development of irritable bowel syndrome: focus on a microbial role. Infect Dis Clin N Am. 2010;24(4):961-975. doi:10.1016/j.idc. 2010.07.005

6. Imperatore N, Tortora R, Morisco F, Caporaso N. Gut microbiota and functional diseases of the gastrointestinal tract. Minerva Gastroenterol Dietol. 2017;63:355-372. doi:10.23736/S1121-421X.16.02336-9

7. Sibelli A, Chalder T, Everitt H, et al. Positive and negative affect mediate the bidirectional relationship between emotional processing and symptom severity and impact in irritable bowel syndrome. $J$ Psychosom Res. 2018;105:1-13. doi:10.1016/j.jpsychores.2017.11.016

8. Radziwon CD, Lackner JM. Cognitive behavioral therapy for ibs: how useful, how often, and how does it work? Curr Gastroenterol Rep. 2017;19(10):49. doi:10.1007/s11894-017-0590-9

9. Wang Y, Chen F, Shi H, et al. Extrinsic ghrelin in the paraventricular nucleus increases small intestinal motility in rats by activating central growth hormone secretagogue and enteric cholinergic receptors. Peptides. 2015;74:43-49. doi:10.1016/j. peptides.2015.09.009

10. Chao G-Q, Zhang S. A meta-analysis of the therapeutic effects of amitriptyline for treating irritable bowel syndrome. Intern Med. 2013;52(4):419-424. doi:10.2169/internalmedicine.52.9147

11. Ford AC, Quigley EM, Lacy BE, et al. Effect of antidepressants and psychologicaltherapies, including hypnotherapy, in irritable bowel syndrome: systematic review and meta-analysis. Am J Gastroenterol. 2014;109:1350-1365. doi:10.1038/ajg.2014.148

12. Xie JF, Shao YF, Wang HL, et al. Neuropeptide S Counteracts Paradoxical Sleep Deprivation-Induced Anxiety-Like Behavior and Sleep Disturbances. Front Cell Neurosci. 2018;12:64. doi:10.3389/ fncel.2018.00064

13. Thomasson J, Canini F, Poly-Thomasson B, et al. Neuropeptide $\mathrm{S}$ overcomes short term memory deficit induced by sleep restriction by increasing prefrontal cortex activity. Eur Neuropsychopharmacol. 2017;27(12):1308-1318. doi:10.1016/j. euroneuro.2017.08.431

14. Duarte-Neves J, Pereira de Almeida L, Cavadas C. Neuropeptide $\mathrm{Y}$ (NPY) as a therapeutic target for neurological diseases. Neurobiol Dis. 2016;95:210-224.

15. Quirk S, Hopkins MM, Bureau H, et al. Mutational analysis of neuropeptide $\mathrm{y}$ reveals unusual thermal stability linked to higher-order self-association. ACS Omega. 2018;3(2):2141-2154. doi:10.1021/acsomega.7b01949

16. Oikonomopoulou K, Hansen KK, Saifeddine M, et al. Kallikreinmediated cell signalling: targeting proteinase-activated receptors (PARs). Biol Chem. 2006;387:817-824. doi:10.1515/BC.2006.104

17. Cenac N, Coelho AM, Nguyen C, et al. Induction of intestinal inflammation in mouse by activation of proteinase-activated receptor-2. Am J Pathol. 2002;161:1903-1915. doi:10.1016/S00029440(10)64466-5

18. Grabauskas G, Wu X, Gao J, et al. Prostaglandin E2, produced by mast cells in colon tissues from patients with irritable bowel syndrome, contributes to visceral hypersensitivity in mice. Gastroenterology. 2020;158(8):2195-2207. doi:10.1053/j.gastro.2020.02.022

19. Guilarte M, Vicario M, Martínez C, et al. Peripheral Corticotropin-Releasing Factor Triggers Jejunal Mast Cell Activation and Abdominal Pain in Patients With Diarrhea-Predominant Irritable Bowel Syndrome. Am J Gastroenterol. 2020;115(12):2047-2059. doi:10.14309/ajg.0000000000000789

20. Leon A, Ceauu Z, Ceauu M, et al. Mast cells and dendritic cells in basal cell carcinoma. Revue Roumaine De Morphologie Et Embryologie. 2009;50:85-90. 
21. Humphreys TR, Monteiro MR, Murphy GF. Mast Cells and Dendritic Cells in Basal Cell Carcinoma Stroma. Dermatologic Surgery. 2010;26:200-204. doi:10.1046/j.1524-4725.2000.09207.x

22. Liu DR, Xu XJ, Yao SK. Increased intestinal mucosal leptin levels in patients with diarrhea-predominant irritable bowel syndrome. World J Gastroenterol. 2018;24:46-57. doi:10.3748/wjg.v24.i1.46

23. Shah K, Maghsoudlou P. Enzyme-linked immunosorbent assay (ELISA): the basics. Br J Hosp Med. 2016;77(7):C98-C101. doi:10.12968/hmed.2016.77.7.C98

24. Liu G, Wang Y, Wang C, et al. Clinical efficacy and safety of apatinib as maintenance treatment in patients with advanced esophageal squamous cell carcinoma. Expert Rev Clin Pharmacol. 2020;13 (12):1423-1430. doi:10.1080/17512433.2020.1844004

25. Gopi K. Prevalence of irritable bowel syndrome in college students and assoctation with anxiety, depression and food map diet. J Mazandaran Univ Med Sci. 2015;2:4949-4957.

26. Elyse T, Brian Q, Hashem E, et al. Medical comorbidity and distress in patients with irritable bowel syndrome: the moderating role of age. J Psychosom Res. 2016;88:48-53. doi:10.1016/j.jpsychores.2016.07.006

27. Kask A, Nguyen HP, Pabst R, et al. Neuropeptide Y Y1 receptor-mediated anxiolysis in the dorsocaudal lateral septum: functional antagonism of corticotropin-releasing hormone-induced anxiety. Neuroscience. 2001;104(3):799-806. doi:10.1016/S03064522(01)00116-6

28. Rizzi A, Vergura R, Marzola G, et al. Neuropeptide S is a stimulatory anxiolytic agent: a behavioural study in mice. Br J Pharmacol. 2008;154(2):471-479. doi:10.1038/bjp.2008.96

29. Zhang L, Riepler SJ, Turner N, et al. Y2 and Y4 receptor signaling synergistically act on energy expenditure and physical activity. Am J Physiol Regul Integr Comp Physiol. 2010;299(6):1618-1628. doi:10.1152/ajpregu.00345.2010

30. Han RW, Chang M, Peng YL, et al. Central Neuropeptide S inhibits distal colonic transit through activation of central Neuropeptide S receptor in mice. Peptides. 2009;30:1313-1317. doi:10.1016/j. peptides.2009.03.012

31. Ann M. Rasmusson. The gut peptide neuropeptide $\mathrm{Y}$ and post-traumatic stress disorder. Curr Opin Endocrinol Diabetes Obes. 2017;24:3-8. doi:10.1097/MED.0000000000000301
32. Tough IR, Forbes S, Tolhurst R, et al. Endogenous peptide YY and neuropeptide $\mathrm{Y}$ inhibit colonic ion transport, contractility and transit differentially via $\mathrm{Y} 1$ and $\mathrm{Y} 2$ receptors. $\mathrm{Br} J$ Pharmacol. 2011;164:471-484. doi:10.1111/j.1476-5381.2011.01401.x

33. Forbes SC, Cox HM, Peptide YY. Neuropeptide Y and corticotrophin-releasing factor modulate gastrointestinal motility and food intake during acute stress. Neurogastroenterol Motil. 2014;26:1605-1614. doi:10.1111/nmo.12428

34. McCoy KL, Traynelis SF, Hepler JR. PAR1 and PAR2 couple to overlapping and distinct sets of $\mathrm{G}$ proteins and linked signaling pathways to differentially regulate cell physiology. Mol Pharmacol. 2010;77(6):1005-1015. doi:10.1124/mol.109.062018

35. Lee KN, Lee OY. The role of mast cells in irritable bowel syndrome. Gastroenterol Res Pract. 2016;2016:2031480. doi:10.1155/2016/ 2031480

36. Bednarska O, Walter SA, Casado-Bedmar M, et al. Vasoactive intestinal polypeptide and mast cells regulate increased passage of colonic bacteria in patients with irritable bowel syndrome. $J$ Gastroenterol. 2017;153(4):948-960. doi:10.1053/j.gastro.2017.06.051

37. Reed DE, Barajas-Lopez C, Cottrell G, et al. Mast cell tryptase and proteinase-activated receptor 2 induce hyperexcitability of guineapig submucosal neurons. J Physiol. 2003;547:531-542. doi:10.1113/ jphysiol.2002.032011

38. Cenac N, Coelho AM, Nguyen C, et al. Induction of intestinal inflammation in mouse by activation of proteinase-activated receptor-2. Am J Pathol. 2002;161:1903-1915.

39. Cenac N, Andrews CN, Holzhausen M, et al. Role for protease activity in visceral pain in irritable bowel syndrome. $J$ Clin Invest. 2007;117(3):636-647. doi:10.1172/JCI29255
Journal of Inflammation Research

\section{Publish your work in this journal}

The Journal of Inflammation Research is an international, peerreviewed open-access journal that welcomes laboratory and clinical findings on the molecular basis, cell biology and pharmacology of inflammation including original research, reviews, symposium reports, hypothesis formation and commentaries on: acute/chronic inflammation; mediators of inflammation; cellular processes; molecular

\section{Dovepress}

mechanisms; pharmacology and novel anti-inflammatory drugs; clinical conditions involving inflammation. The manuscript management system is completely online and includes a very quick and fair peerreview system. Visit http://www.dovepress.com/testimonials.php to read real quotes from published authors. 